\title{
SOME COMPUTATIONAL PROBLEMS RELATED TO NORMAL FORMS
}

\author{
VU DUC THI
}

\begin{abstract}
In the relational database theory the most desirable normal form is the BoyceCodd normal form (BCNF). This paper investigates some computational problems concerning $\mathrm{BCNF}$ relation scheme and $\mathrm{BCNF}$ relations. We give an effective algorithm finding a $\mathrm{BCNF}$ relation $\mathrm{r}$ such that $\mathrm{r}$ represents a given $\mathrm{BCNF}$ relation scheme $s$ (i.e., $K_{r}=K_{s}$, where $K_{r}$ and $K_{s}$ are sets of all minimal keys of $r$ and $s$ ). This paper also gives an effective algorithm which from a given BCNF relation finds a BCNF relation scheme such that $K_{r}=K_{s}$. Based on these algorithms we prove that the time complexity of the problem that finds a BCNF relation $r$ representing a given BCNF relation scheme $s$ is exponential in the size of $s$ and conversely, the complexity of finding a BCNF relation scheme $s$ from a given BCNF relation $r$ such that $r$ represents $s$ also is exponential in the number of attributes.

We give a new characterization of the relations and the relation schemes that are uniquely determined by their minimal keys. It is known that these relations and the relation schemes are in the BCNF class. From this characterization we give a polynomial time algorithm deciding whether an arbitrary relation is uniquely determined by its set of all minimal keys. In the rest of this paper some new bounds of the size of minimal Armstrong relations for BCNF relation scheme are given. We show that given a Sperner system $K$ and BCNF relation scheme $s$ a set of minimal keys of which is $K$, the number of antikeys (maximal nonkeys) of $K$ is polynomial in the number of attributes iff so is the size of minimal Armstrong relation of $s$.
\end{abstract}

Key words and phrases:

Relation, relational, datamodel, functional dependency, relation scheme, Boyce-Codd normal form, closure, closed set, minimal generator, key, minimal key, antikey.

\section{INTRODUCTION}

In the relation datamodel, one of the important concepts is the functional dependency (FDs). Several types of families of FDs which satisfy some conditions are known under the name normal forms (NFs). The Boyce-Codd normal form has been investigated in a lot of papers. It is shown [4] that every set of attributes with an associated set of FDs has a decomposition into third NF which has the loss-less-join property and preserves FDs. However, for BCNF this doesn't always exists. The key is an interesting concept in the relational datamodel. In this 
paper we present some computational problem related to connections between sets of minimal keys, relation schemes and relations in BCNF class.

Let us give some necessary definitions and results that are used in the next section.

Definition 1. Let $R=\left\{a_{1}, \ldots, a_{n}\right\}$ be a nonempty finite set of attributes, $r=$ $\left\{h_{1}, \ldots, h_{m}\right\}$ be a relation over $R$, and $A, B \subseteq R$

Then we say that $B$ functionally depends on $A$ in $r$ (denoted $A \underset{r}{\stackrel{f}{\rightarrow}} B$ ) iff

$$
\left(\forall h_{i}, h_{j} \in r\right)\left((\forall a \in A)\left(h_{i}(a)=h_{j}(a)\right) \Rightarrow(\forall b \in B)\left(h_{i}(b)=h_{j}(b)\right) .\right.
$$

Let $F_{r}=\{(A, B): A, B \subseteq R, A \underset{r}{\stackrel{f}{\rightarrow}} B\} . F_{r}$ is called the full family of functional dependencies of $r$. Where we write $(A, B)$ or $A \rightarrow B$ for $A \underset{r}{\stackrel{f}{\rightarrow}} B$ when $r, f$ are clear from the context.

Definition 2. A functional dependency over $R$ is a statement of the form $A \rightarrow B$, where $A, B \subseteq R$. The FD $A \rightarrow B$ holds in a relation $r$ if $\underset{r}{\stackrel{f}{\rightarrow}} B$. We also say that $r$ satisfies the FD $A \rightarrow B$.

Clearly, $F_{r}$ is a set of all FDs that hold in $r$.

Definition 3. Let $R$ be a nonempty finite set, and denote $P(R)$ its power set. Let $y \subseteq P(R) \times P(R)$. We say that $y$ is an $f$ family over $R$ iff for all $A, B, C, D \subseteq R$

(1) $(A, A) \in y$,

(2) $(A, B) \in y,(B, C) \in y \Rightarrow(A, C) \in y$,

(3) $(A, B) \in y, A \subseteq C, D \subseteq B \Rightarrow(C, D) \in y$,

(4) $(A, B) \in y,(C, D) \in y \Rightarrow(A \cup C, B \cup D) \in y$.

Clearly $F_{r}$ is an $f$-family over $R$.

It is known [1] that if $y$ is an arbitrary $f$-family, then there is a relation $r$ over $R$ such that $F_{r}=y$.

Definition 4. A relation scheme $s$ is a pair $\langle R, E\rangle$, where $R$ is a set of attributes, and $F$ is a set of FDs over $R$. Let $F^{+}$be a set of all FDs that can be derived from $F$ by the rules in Definition 3. Denote $A^{+}=\left\{a: A \rightarrow\{a\} \in F^{+}\right\} . A^{+}$is called the closure of $A$ over s. It is clear that $A \rightarrow B \in F^{+}$iff $B \subseteq A^{+}$.

Clearly, if $s=\langle R, F\rangle$ is a relation scheme, then there a relation $r$ over $R$ such that $F_{r}=F^{+}$(see [1]). Such a relation is called an Armstrong relation of $s$. It is obvious that all FDs of $s$ hold in $r$.

Definition 5. Let $r$ be a relation, $s=\langle R, F\rangle$ be a relation scheme, $y$ be an 
$f$-family over $R$ and $A \subseteq R$. Then $A$ is a key of $r$ (a key of $s$, a key of $y$ ) if $A \underset{r}{\stackrel{f}{\rightarrow}} R$ $\left(A \rightarrow R \in F^{+},(A, R) \in y\right) . A$ is a minimal key of $r(s, y)$ if $A$ is a key of $r(s, y)$, and any proper subset of $A$ is a key of $r(s, y)$. Denote $K_{r}\left(K_{s}, K_{y}\right)$ the set of all minimal keys of $r(s, y)$.

Clearly, $K_{r}, K_{s}, K_{y}$ are Sperner systems over $R$ (i.e. $A, B \in K_{r}$ implies $A \nsubseteq B)$.

Definition 6. Let $K$ be a Sperner system over $R$. We define the set of antikeys of $K$, denoted by $K^{-1}$, as follows:

$$
K^{-1}=\{A \subset R:(B \in K) \Rightarrow(B \nsubseteq \mathbb{E}) \text { and }(A \subset C) \Rightarrow(\exists B \in K)(B \subseteq C)\} .
$$

It is easy to see that $K^{-1}$ is also a Sperner system over $R$.

It is known [6] that if $K$ is an arbitrary Sperner system over $R$ then there is a relation scheme $s$ such that $K_{s}=K$.

In this paper we always assume that if a Sperner system plays the role of the set of minimal keys (antikeys), then this Sperner system is not empty of algorithms. Thus, if we assume that subsets of $R$ are represented as sorted lists of attributes, then a Boolean operation on two subsets of $R$ requires at most $|R|$ elementary steps.

Definition 7. Let $I \subseteq P(R), R \in I$, and $A, B \in I \Rightarrow A \cap B \in I$. Let $M \subseteq P(R)$. Denote $M^{+}=\left\{M^{\prime}: M^{\prime} \subseteq M\right\}$. We say that $M$ is a generator of $I$ iff $M^{+}=I$. Note that $R \in M^{+}$but not in $M$, since it is the intersection of the empty collection of sets.

Denote $N=\left\{A \in I: A \neq \cap\left\{A^{\prime} \in I: A \subset A^{\prime}\right\}\right\}$.

In [6] it is proved that $N$ is the unique minimal generator of $I$. Thus, for any generator $N^{\prime}$ of $I$ we obtain $N \subseteq N^{\prime}$.

Definition 8. Let $r$ be a relation over $R$, and $E_{r}$ the equality set of $r$, i.e. $E_{r}=\left\{E_{i j}: 1 \leq i<j \leq|r|\right\}$, where $E_{i j}=\left\{a \in R: b_{i}(a)=b_{j}(a)\right\}$. Let $T_{r}=\left\{A \in P(R): \exists E_{i j}=A, \nexists E_{p q}: A \subset E_{p q}\right\}$. Then $T_{r}$ is called the maximal equality system of $r$.

Definition 9. Let $r$ be a relation, and $K$ a Sperner system over $R$. We say that $r$ represents $K$ iff $K_{r}=K$.

The following theorem is known ([8]).

Theorem 1. Let $K$ be a non-empty Sperner system and $r$ a relation over $R$. Then $r$ represents $K$ iff $K^{-1}=T_{r}$, where $T_{r}$ is the maximal equality system of $r$. 
Let $s=\langle R, F\rangle$ be a relation scheme over $R, K_{s}$ is a set of all minimal keys of $s$. Denote $K_{s}^{-1}$ the set of all antikeys of $s$. From Theorem 1 we obtain the following corollary.

Corollary 1. Let $s=\langle R, F\rangle$ be a relation scheme and $r$ a relation over $R$. We say that $r$ represents $s$ if $K_{r}=K_{s}$. Then $r$ represents $s$ iff $K_{s}^{-1}=T_{r}$ is the maximal equality system of $r$.

In [7] we proved the following theorem.

Theorem 2. Let $r=\left\{h_{1}, \ldots, h_{m}\right\}$ be a relation, and $F$ an $f$-family over $R$. Then $F_{r}=F$ iff for every $A \in P(R)$

$$
H_{F}(A)= \begin{cases}\bigcap_{A \subseteq A_{i j}} E_{i j} & \text { if } \exists E_{i j} \in E_{r}: A \subseteq E_{i j} \\ R & \text { otherwise }\end{cases}
$$

where $H_{F}(A)=\{a \in R:(A,\{a\}) \in F\}$ and $E_{r}$ is the equality set of $r$.

We say that a relation scheme $s=\langle R, F\rangle$ (a relation $r$ ) is in BCNF if $\forall A \subseteq R$ either $A^{+}=A$ or $A^{+}=R\left(H_{F_{r}}(A)=A\right.$ or $\left.H_{F_{r}}(A)=R\right)$.

\section{RESULTS}

All relation and relation schemes investigated in this section are in BCNF. First we construct two combinatorial algorithms concerning minimal keys of relations and relation schemes. We estimate these algorithms. After that we presents two problems the worst-case time complexity of which are exponential.

Let $s=\langle R, F\rangle$ be a relation scheme over $R$. From $s$ we construct $Z(s)=$ $\left\{X^{+}: X \subseteq R\right\}$, and compute the minimal generator $N_{s}$ of $Z(s)$. We put

$$
T_{s}=\left\{A \in N_{s}: \nexists B \in N_{s}: A \subset B\right\}
$$

It is known [1] that for a given relation scheme $s$ there is a relation $r$ such that $r$ is an Armstrong relation of $s$. On the other hand, by Corollary 1 and Theorem 2 the following proposition is clear.

Proposition 1. Let $s=\langle R, F\rangle$ be a relation scheme over $R$. Then

$$
K_{s}^{-1}=T_{s} .
$$


Definition 10. Let $s=\langle R, F\rangle$ be a relation scheme. We say that $s$ is a $k$-relation scheme over $R$ if $F=\left\{K_{1} \rightarrow R, \ldots, K_{m} \rightarrow R\right\}$, where $\left\{K_{1}, \ldots, K_{m}\right\}$ is a Sperner system over $R$. It is easy to see that $K_{s}=\left\{K_{1}, \ldots, K_{m}\right\}$.

Clearly, if $s=\langle R, F\rangle$ is in BCNF then using the algorithm for finding a minimal cover in poly nomial time we can construct a $k$-relation scheme $s^{\prime}=\left\langle R, F^{\prime}\right\rangle$ such that $F^{+}=F^{\prime+}$, see $[10]$. Conversely, it can be seen that an arbitrary $k$ relation scheme is in $\mathrm{BCNF}$. Consequently, we can consider a relation scheme in $\mathrm{BCNF}$ as a $k$-relation scheme.

Remark 1. It is known [10] that $s=\langle R, F\rangle$ is in BCNF iff its minimum cover is a $k$-relation scheme. Consequently, the BCNF property of $s$ is polynomially recognizable.

Let $r$ be a relation over $R$. From $r$ we compute $E_{r}$. We construct the maximal equality system $T_{r}$ of $r$. By Theorem 1 we obtain $T_{r}=K_{r}^{-1}$. Denote elements of $T_{r}$ by $A_{1}, \ldots, A_{t}$.

Set $M_{r}=\left\{A_{i}-a: a \in R, i=1, \ldots, t\right\}$. Denote elements of $M_{r}$ by $B_{1}, \ldots, B_{s}$. We construct the relation $r^{\prime}=\left\{h_{0}, h_{1}, \ldots, h_{s}\right\}$ as follows:

For all $a \in R, h_{0}(a)=0$, for each $i=1, \ldots, s h_{i}(a)=0$ if $a \in B_{i}$, in the converse case we set $h_{i}(a)=i$.

By [10] $r^{\prime}$ is BCNF and $K_{r}=K_{r^{\prime}}(1)$. It is easy to see that $M_{r}$ and $r^{\prime}$ are constructed in polynomial time in the size of $r$.

Set $H_{F_{r}}(A)=\left\{a \in R:(A,\{a\}) \in F_{r}\right\}, Z_{F_{r}}=\left\{A: H_{F_{r}}(A)=A\right\}$. Denote by $N_{F_{r}}$ the minimal generator of $Z_{F_{r}}$.

Based on definition of BCNF relation and from (1) we can see that a relation $r$ is in BCNF iff $N_{F_{r}}=N_{F_{r}}^{\prime}$. Because for an arbitrary relation $r N_{F_{r}}$ is computed in polynomial time, the BCNF property of $r$ can be tested in polynomial time.

We give the following algorithm that from a given relation scheme $s$ constructs a relation $r$ such that $r$ represents $s$.

It is known [15] that there is an algorithm that finds a set of all antikeys from a given Sperner system.

Algorithm 1 (Finding a set of antikeys).

Input: Let $K=\left\{B_{1}, \ldots, B_{m}\right\}$ be a Sperner system over $R$.

Output: $K^{-1}$.

Step1: We set $K_{1}=\left\{R-\{a\}: a \in B_{1}\right\}$. It is obvious that $K_{1}=\left\{B_{1}\right\}^{-1}$.

Step $q+1(q<m)$ : We assume that $K_{q}=F_{q} \cup\left\{X_{1}, \ldots, X_{t_{q}}\right\}$, where $X_{1}, \ldots, X_{t_{q}}$ containing $B_{q+1}$ and $F_{q}=\left\{A \in K_{q}: B_{q+1} \notin A\right\}$. For all $i\left(i=1, \ldots, t_{q}\right)$ we construct the antikeys of $\left\{B_{q+1}\right.$ on $X_{i}$ in an analogous way as $K_{1}$. Denote them by $A_{1}^{i}, \ldots, A_{r_{i}}^{i}\left(i=1, \ldots, t_{q}\right)$. Let 


$$
K_{q+1}=F_{q} \cup\left\{A_{p}^{i}: A \in F_{q} \Rightarrow A_{p}^{i} \notin A, 1 \leq i \leq t_{q}, 1 \leq p \leq r_{i}\right\} .
$$

We set $K^{-1}=K_{m}$.

Theorem $3[15]$. For every $q(1 \leq q \leq m), K_{q}=\left\{B_{1}, \ldots, B_{q}\right\}^{-1}$, i.e. $K_{m}=K^{-1}$.

It can be seen that $K$ and $K^{-1}$ are uniquely determined by one another and the determination of $K^{-1}$ based on our algorithm does not depend on the order of $B_{1}, \ldots, B_{m}$. Denote $K_{q}=F_{q} \cup\left\{X_{1}, \ldots, X_{t_{q}}\right\}$ and let $l_{q}(1 \leq q \leq m-1)$ be the number of elements of $K_{q}$.

Proposition 2 [15]. The worst-case time complexity of Algorithm 1 is

$$
O\left(|R|^{2} \sum_{q=1}^{m-1} l_{q} u_{q}\right)
$$

where

$$
u_{q}= \begin{cases}l_{q}-t_{q} & \text { if } l_{q}>t_{q} \\ 1 & \text { if } l_{q}=t_{q}\end{cases}
$$

Clearly, in each step of our algorithm $K_{q}$ is a Sperner system. In the cases for which $l_{q} \leq l_{m}(q=1, \ldots, m-1)$ it is easy to see that the time complexity of our algorithm is not greater than $O\left(|R|^{2}|K|\left|K^{-1}\right|^{2}\right)$. Thus, in these cases Algorithm 1 finds $K^{-1}$ in polynomial time in $|R|,|K|$, and $\left|K^{-1}\right|$. It can be seen that if the number of elements of $K$ is small then Algorithm 1 is very effective. It only requires polynomial time in $|R|$.

By Algorithm 1 we construct an algorithm that finds a relation such that this relation represents a given relation scheme. By Remark 1 it is simple that we can consider an arbitrary relation scheme in BCNF as a $k$-relation scheme.

\section{Algorithm 2.}

Input: $s=\left\langle R, F=\left\{K_{1} \rightarrow R, \ldots, K_{m} \rightarrow R\right\}\right\rangle$ be a $k$-relation scheme.

Output: A BCNF relation $r$ such that $K_{r}=K_{s}$.

Step 1: From $K=\left\{K_{1}, \ldots, K_{m}\right\}$ we construct $K^{-1}=\left\{B_{1}, \ldots, B_{t}\right\}$ by Algorithm 1 .

Step 2: Set $M=\left\{B_{i}-a: a \in R, i=1, \ldots, t\right\}$.

Step 3: Denote elements of $M$ by $A_{1}, \ldots, A_{t}$, construct a relation $r=\left\{h_{0}, h_{1}, \ldots, i\right.$ as follows: For all $a \in R: h_{0}(a)=0$. For $i=1, \ldots, l$ we set $h_{i}(a)=0$ if $a \in A_{i}$, in the converse case $h_{i}(a)=i$.

By Remark 1 , Corollary 1 we obtain $K_{r}=K_{s}$, and $r$ is a BCNF relation. 
Clearly, set $M$ and relation $r$ are constructed in polynomial time in the size of $K^{-1}$. Consequently, the time complexity of this algorithm is $O\left(|R|^{3} \sum_{q=1}^{m-1} l_{q} u_{q}\right)$, for meanings of $t_{q}, u_{q}$ see Proposition 2. In many cases this algorithm requires polynomial time in the size of $s$ (see Proposition 2).

Now we construct an algorithm which finds a BCNF relation scheme such that a given $\mathrm{BCNF}$ relation represents this relation scheme. First we give the following algorithm.

Algorithm 3. [8] (Finding a minimal key from a set of antikeys)

Input: Let $K$ be a Sperner system, $H$ a Sperner system, and $C=\left\{b_{1}, \ldots, b_{m}\right\} \subseteq$ $R$ such that $H^{-1}=K$ and $\exists B \in K: B \subseteq C$.

Output: $D \in H$.

Step 1: Set $T(0)=C$.

Step i+1: Set $T=T(i)-b_{i+1}$

$$
T_{i+1}= \begin{cases}T & \text { if } \forall B \in K: T \nsubseteq B \\ T(i) & \text { otherwise }\end{cases}
$$

We set $D=T(m)$.

Lemma 1 [8]. If $K$ is a set of antikeys, then $T(m) \in H$.

Lemma $2[8]$. Let $H$ be a Sperner system over $R$, and $H^{-1}=\left\{B_{1}, \ldots, B_{m}\right\}$ be a set of antikeys of $H, T \subseteq H$. Then $T \subset H, T \neq \emptyset$ if and only if there is $a B \subseteq R$ such that $B \in T^{-1}, B \notin B_{i}(\forall i: 1 \leq i \leq m)$.

Based on Lemma 2, Algorithm 3 we have the following algorithm.

Algorithm 4. (Finding a set of minimal keys from a set of antikeys)

Input: Let $K=\left\{B_{1}, \ldots, B_{k}\right\}$ be a Sperner system over $R$.

Output: $H$ such that $H^{-1}=K$.

Step 1: By Algorithm 3 we compute an $A_{1}$, set $K(1)=A_{1}$.

Step $\mathrm{i}+1$ : If there exists a $B \in K_{i}^{-1}$ such that $B \nsubseteq B_{j}(\forall j: 1 \leq j \leq k)$, then by Algorithm 3 we compute an $A_{i+1}$, where $A_{i+1} \in H, A_{i+1} \subseteq B$. Set $K(i+1)=K(i) \cup A_{i+1}$. In the converse case we set $H=K(i)$.

Proposition $3[16]$. The time complexity of Algorithm \& is $O\left(n\left(\sum_{q=1}^{m-1}\left(k l_{q}+\right.\right.\right.$ $\left.\left.\left.n t_{q} u_{q}\right)+k^{2}+n\right)\right)$, where $|R|=n,|K|=k,|H|=m$, for meanings of $l_{q}, t_{q}, u_{q}$ see Proposition 2. 
Clearly, in cases for which $l_{q} \leq k(\forall q: 1 \leq q \leq m-1)$ the time complexity of our algorithm is $O\left(|R|^{2}|K|^{2}|H|\right)$. It is easy to see that in these cases Algorithm 4 finds the set of minimal keys in polynomial time in the sizes of $R, K, H$. If $|H|$ is polynomial in $|R|$ and $|K|$, then our algorithm is effective. It can be seen that if the number of elements of $H$ is small then Algorithm 4 is very effective.

\section{Algorithm 5.}

Input: Let $r$ be a BCNF relation over $R$.

Output: A BCNF relation scheme $s$ such that $K_{s}=K_{r}$.

Step 1: From $r$ compute $E_{r}$.

Step 2: From $E_{r}$ compute the maximal equality system $T_{r}$.

Step 3: By Algorithm 4 we construct a set of all minimal keys $H$ of $r$.

Step 4: Denoting elements of $H$ by $A_{1}, \ldots, A_{m}$ we construct a relation scheme as follows: $s=\langle R, F\rangle$, where $F=\left\{A_{1} \rightarrow R, \ldots, A_{m} \rightarrow R\right\}$.

Based on Theorem 1, Algorithm 4 and Definition 10 we have $K_{s}=K_{r}$. It is clear that the time complexity of this algorithm is the time complexity of Algorithm 4. In many case this algorithm is very effective (see Proposition 3).

Theorem 4 [14]. Let $K$ be a Sperner system over $R$. Denote $s(K)=\min \{m$ : $\left.|r|=m, K_{r}=K\right\}$. Then $\left(2\left|K^{-1}\right|\right)^{1 / 2} \leq s(K) \leq\left|K^{-1}\right|+1$.

Remark 2. Let us take a partition $R=X_{1} \cup \cdots \cup X_{m} \cup W$, where $|R|=n$, $m=[n / 3]$, and $\left|X_{i}\right|=3(1 \leq i \leq m)$.

We set

$H=\left\{B:|B|=2, B \subseteq X_{i}\right.$ for some $\left.i\right\}$ if $|W|=O$.

$H=\left\{B:|B|=2, B \subseteq X_{i}\right.$ for some $i: 1 \leq i \leq m-1$ or $\left.B \subseteq X_{m} \cup W\right\}$ if $|W|=1$,

$H=\left\{B:|B|=2, B \subseteq X_{i}\right.$ for some $i: 1 \leq i \leq m$ or $\left.B=W\right\}$ if $|W|=2$.

It is easy to see that

$H^{-1}=\left\{A:\left|A \cap X_{i}\right|=1, \forall i\right\}$ if $|W|=0$.

$H^{-1}=\left\{A:\left|A \cap X_{i}\right|=1(1 \leq i \leq m-1)\right.$ and $\left.\left|A \cap\left(X_{m} \cup W\right)\right|=1\right\}$ if $|W|=1$,

$H^{-1}=\left\{A:\left|A \cap X_{i}\right|=1(1 \leq i \leq m)\right.$ and $\left.|A \cap W|=1\right\}$ if $|W|=2$.

If set $K=H^{-1-1}$, i.e. $H^{-1}$ is a set of minimal keys of $K$, then we have

$K=\left\{C:|C|=n-3, C \cap X_{i}=\emptyset\right.$ for some $\left.i\right\}$ if $|W|=0$,

$K=\left\{C:|C|=n-3, C \cap X_{i}=\emptyset\right.$ for some $i(1 \leq i \leq m-1)$ or $|C|=n-4$, $\left.C \cap\left(X_{m} \cup W\right)=\emptyset\right\}$ if $|W|=1$,

$K=\left\{C:|C|=n-3, C \cap X_{i}=\emptyset\right.$ for some $i(1 \leq i \leq m)$ or $|C|=n-2$, $C \cap W=\emptyset\}$ if $|W|=2$. 
It is clear that $n-1 \leq|H| \leq n+2,3^{[n / 4]} \leq\left|H^{-1}\right|,|K| \leq m+1$. Based on this partition, Theorem 4 and algorithms 2, 5 we obtain the following theorem.

Theorem 5. In the BCNF class of and relation schemes, the time complexity of finding a relation $r$ from a given relation scheme $s$ such that $r$ represents $s$ is exponential in the size of $s$.

Proof: We have to prove that:

(1) There is an algorithm finding a BCNF relation $r$ from a given $\mathrm{BCNF}$ relation scheme $s$ such that $r$ represents $s$ and the time complexity of this algorithm is exponential time in the size of $s$.

(2) There exists a $\mathrm{BCNF}$ relation scheme $s=\langle R, F\rangle$ such that the number of rows of any BCNF relation representing $s$ is exponential in the size of $s$.

For (1): We have Algorithm 2.

For (2): According to Theorem 4 we have $\left(2 \mid K^{-1}\right)^{1 / 2} \leq s(K)$. We construct a $k$-relation scheme $s=\langle R, F\rangle$, where $F=\{B \rightarrow R: B \in H\}$. It is obvious that $H^{-1}=K_{s}^{-1}$. Hence, $\left(2^{1 / 2} 3^{[n / 8]}\right) \leq s\left(K_{s}\right)$ holds. It can be seen that BCNF relation $r$ that is constructed in Algorithm 2 has the number of rows at most $|U|\left|H^{-1}\right|+1$. Thus, we always can construct a BCNF relation scheme $s$ such that the number of rows of any $\mathrm{BCNF}$ relation representing $s$ is exponential in the size of $s$. The proof is complete.

Theorem 6. In BCNF class of relations and relation schemes over $R$, the time complexity of finding a relation scheme s from a given relation $r$ such that $K_{r}=K_{s}$ is exponential in the number of attributes.

Proof: It is clear that the worst-case time complexity of Algorithm 5 is exponential in the size of $R$. In Remark 2 we have $|K| \leq m+1$. We set $M=\{C-a: \forall a, C$ : $a \in R, C \in K\}$. Denote elements of $M$ by $C_{1}, \ldots, C_{t}$. Construct a relation $r=\left\{h_{0}, h_{1}, \ldots, h_{t}\right\}$ as follows: For all $a \in R h_{0}(a)=0$. For $i=1, \ldots, t h_{i}(a)=0$ if $a \in C_{i}$, in the converse case $h_{i}(a)=i$. Clearly, $|r| \leq(m+1)|R|+1$ holds. We construct a relation scheme $s=\langle R, F\rangle$ with $F=\left\{A \rightarrow R: A \in H^{-1}\right\}$. It is obvious that $3^{[n / 4 !} \leq|F|$, and $K_{r}=K_{s}$. Clearly, a minimum cover of any BCNF relation scheme is a $k$-relation scheme. Thus, we always can construct a $\mathrm{BCNF}$ relation scheme $s=\langle R, F\rangle$ such that $K_{r}=K_{s}$. The.number of elements of $F$ is exponential in the number of attributes. Our proof is complete.

It is known that in the BCNF class a relation $r$ represents a relation scheme $s$ iff $r$ is an Armstrong relation of $s$. Consequently, our two algorithms and two problems are still true when $r$ is an Armstrong relation of $s$.

Proposition 4. Let $s=\langle R, F\rangle$ be a relation scheme. Then $s$ is in $B C N F$ iff $\forall B \in K_{s}^{-1}, a \in B:(B-a)^{+}=B-a$. 
Proof: It is easy to see that if $s$ is in BCNF then $(B-a)^{+}=B-a$ for $B \in K_{s}^{-1}$ and $a \in B$.

Conversely, assume that $s$ is not in BCNF. Consequently, there is an $A \rightarrow$ $\{a\} \in F^{+}$, where $A^{+} \neq R$ and $a \notin A$. By Proposition 1 there is a $B \in K_{s}^{-1}$ such that $A^{+} \subseteq B$. Clearly, $a \in B$ and $A \subseteq B-a$. Hence, $(B-a)^{+}=B$ holds. The proof is complete.

Proposition 4 was independently discovered in [17].

Definition 11. Let $K$ be a Sperner system over $R$. We say that $K$ is unique if $K$ uniquely determines a relation scheme $s=\langle R, F\rangle$, i.e. for every relation scheme $s^{\prime}=\left\langle R, F^{\prime}\right\rangle$ such that $K_{s^{\prime}}=K$ we have $F^{+}=F^{\prime+}$.

It is easy to see that $s$ is a BCNF relation scheme.

It is known [6] that for give a Sperner system $K$ there exists a relation scheme $s$ (a relation $r$, respectively) is unique if $K_{s}=K\left(K_{r}=K\right.$, respectively). We say that $s$ ( $r$, respectively) is unique if $K_{s}\left(K_{r}\right.$, respectively) uniquely determines $s$ ( $r$, respectively) i.e. $K_{s}\left(K_{r}\right.$, respectively) is unique.

Now we give a necessary and sufficient condition for a given relation scheme to be unique.

Theorem 7. Let $s=\langle R, F\rangle$ be a relation scheme over $R$. Then $s$ is unique iff for all $a \in A, A \in K_{s}^{-1}: A-a=\cap\left\{B \in K_{s}^{-1}:(A-a) \subset B\right\}$ holds.

Proof: It is known [5] that a Spernersystem $K$ is unique iff for all $B \subseteq A, A \in K^{-1}$, $B$ is an intersection of antikeys. Denote $P_{s}=\left\{A-a: A \in K_{s}^{-1}, a \in A\right\}$.

It can be seen that if $s=\langle R, F\rangle$ is unique then $B \in P_{s}$ implies $B$ is an intersection of antikeys, i.e. $B=\cap\left\{A \in K_{s}^{-1}: B \subseteq A\right\}$.

Conversely, assume that for every $B \in P_{s}$ we have $B=\cap\left\{A \in K_{s}^{-1}: B \subseteq A\right\}$ $(*)$. By Proposition 4 and according to Proposition 1 we have $N_{s} \subseteq\left(P_{s} \cup K_{s}^{-1}\right)$. It can be seen that $s$ in BCNF. Based on definition of $N_{s}$ and Proposition 1 $K_{s}^{-1} \subseteq N_{s}$ holds. According to (*) we obtain $K_{s}^{-1}=N_{s}$. Because $s$ is in BCNF we can see that for all $B \subseteq A, A \in K_{s}^{-1}: B^{+}=B$ holds. Thus, $B$ is an intersection of antikeys of $s$. The proof is complete.

By a polynomial time algorithm finding a set of all antikeys of a given relation (see [14]) and according to Theorem 7 we obtain the followwing proposition.

Proposition 5. There exists an algorithm deciding whether a given relation $r$ is unique. The time complexity of this algorithm is polynomial in the size of $R$ and $r$.

Theorem 7 immediately implies. 
Corollary 2. Let $K$ be a Sperner system over $R$. Then there exists a polynomial time algorithm deciding whether a Sperner system $H$ is unique, where $H^{-1}=K$.

The size of minimal Armstrong relation was investigated in some papers (see $[2,5,6,12,14])$. Now we present some new bounds for the size of Armstrong relation.

Definition 12 (Minimal Armstrong relation). Let $F$ be an $f$-family over $R$. Let $M(F)=\min \left\{m:|r|=m, F_{r}=F\right\}$.

Denote $H_{F}(A)=\{a \in R:(A,\{a\}) \in F\}$, and $Z(F)=\left\{A: H_{F}(A)=A\right\}$.

Proposition 6 [6]. Let $F$ be an $f$-family over $R$. Then

$$
(2|N(F)|)^{1 / 2}<M(F) \leq|N(F)|+1,
$$

where $N(F)$ is the minimal generator of $Z(F)$.

According to the definition of unique Sperner system and based on Theorem 7 we have the following.

Proposition 7. Let $s=\langle R, F\rangle$ be a relation scheme. Then if $K_{s}$ is unique then

$$
\left(\left|K_{s}^{-1}\right|\right)^{1 / 2}<M\left(F^{+}\right) \leq\left|K_{s}^{-1}\right|+1 .
$$

Theorem 8. Let $K$ be a Sperner system, $s=\langle R, F\rangle$ a BCNF relation scheme over $R$. Let

$$
\begin{aligned}
& M\left(F^{+}\right)=\min \left\{m:|r|=m, F_{r}=F^{+}, s=\langle R, F\rangle \text { a relation scheme }\right\} ; \\
& K^{*}=K^{-1} \cup\left\{B-a: B \in K^{-1}, a \in B, B-a \neq \cap\left\{C: C \in K^{-1}, B-a \subset C\right\}\right\} . \\
& \text { Then if } K_{s}=K \text { then }\left(2\left|K^{*}\right|\right)^{1 / 2}<M\left(F^{+}\right) \leq\left|K^{*}\right|+1(*) .
\end{aligned}
$$

Proof: For an arbitrary Sperner system $K$ we can construct a BCNF relation scheme $s=\langle R, F=\{A \rightarrow R: A \in K\}\rangle$ such that $K_{s}=K$. According to definition of BCNF relation scheme we can see that for all BCNF relation scheme $s^{\prime}=\left\langle R, F^{\prime}\right\rangle$ such that $K_{s^{\prime}}=K, F^{+}=F^{\prime+}$ holds.

Denoting the elements of $K^{*}$ by $A_{1}, \ldots, A_{t}$ we construct a relation $r=\left\{h_{0}, h_{1}, \ldots, h_{t}\right\}$ as follows:

For all $a \in R, h_{0}(a)=0 . \forall i=1, \ldots t$

$$
h_{i}(a)= \begin{cases}0 & \text { if } a \in A \\ i & \text { otherwise }\end{cases}
$$

By Proposition 4 we obtain $K_{r}=K$ and $r$ is in BCNF. According to defintion of BCNF we have $F_{r}=F^{+}$. Hence, $N\left(F_{r}\right)=N\left(F^{+}\right)$holds. According to Theorem 
2 we have $N\left(F_{r}\right) \subseteq E_{r}$. Based on Proposition 4 and the construction of $K^{*}$, and $N\left(F_{r}\right)=\left\{B \in E_{r}: B \neq \cap\left\{B^{\prime} \in E_{r}: B \subset B^{\prime}\right\}\right\}$, we obtain $N\left(F_{r}\right)=K^{*}$. According to Proposition 6 we have $\left(2\left|K^{*}\right|\right)^{1 / 2}<M\left(F^{+}\right) \leq\left|K^{*}\right|+1$. The proof is complete.

Let $K$ be a Sperner system over $R$. We say that an attribute a is prime of $K$ if it belongs to an element of $K$, and nonprime otherwise.

Based on Proposition 7 and Theorem 8 we obtain the following.

Proposition 8. Let $K$ be a Sperner system over $R=\left\{a_{1}, \ldots, a_{n}\right\}$. Let $K^{-1}=$ $\left\{A_{1}, \ldots, A_{k}\right\}$ and $K_{n}=\left\{a_{i_{1}}, \ldots, a_{i_{s}}\right\}$ be a set of nonprime attributes of $K$. Then for every $B C N F$ relation scheme $s=\langle R, F\rangle$ such that $K_{s}=K$ then

$$
k(s+1) \leq\left|N\left(F^{+}\right)\right| \leq k n(* *)
$$

Proof: Clearly, if $B \in K^{-1}$ then $|B| \leq n-1$. Hence, in Theorem $8\left|K^{*}\right| \leq k n$. By the proof of Theorem $8 K^{*}=N\left(F^{+}\right)$holds. Thus, $N\left(F^{+}\right) \leq k n$ holds. It is known [7] that $K_{n}$ is the intersection of elements of $K^{-1}$. According to definition of minimal generator we can see that if $B \in K^{-1}$ then for every $a \in$ $K_{n}: B-a \in N\left(F^{+}\right)$. Clearly, if $B \in K^{-1}$ then $B \in N\left(F^{+}\right)$. Consequently, we have $k(s+1) \leq\left|N\left(F^{+}\right)\right| \leq k n$. The proof is complete.

According to Theorem 8 and Proposition 8 we can see that for all BCNF relation scheme $s=\langle R, F\rangle$ such that $K_{s}=K,\left|K^{-1}\right|$ is polynomial in the number of attributes if and only if the size of minimal Armstrong relation and the number of elements of the minimal generator $N\left(F^{+}\right)$(sometimes it is denoted by $\operatorname{GEN}(s)$ ) of $s$ also polynomial in $|R|$.

It can be seen that the bounds $(*)$ and $(* *)$ are especially interesting when the number of antikeys of antikeys of $K$ is polynomial in the number of attributes.

\section{REFERENCES}

1. Armstrong W.W., Dependency Structures of Database Relationships, Information Processing, Holland publ. Co., 74 (1974), 580-583.

2. Beeri C., Dowd M., Fagin R., Staman R., On the Structure of Armstrong relations for Functional Dependencies, J. ACM., 31, 1 (1984), 30-46.

3. Beeri C., Bernstein P.A., Computational problems related to the design of normal form relational schemas, ACM Trans. on Database Syst., 4 (1) (1979), $30-59$.

4. Bernstein P. A., Synthesizing third normal form relations from functional dependencies, ACM Trans. Database Systems, 1 (1976), 227-298.

5. Burosch G., Demetrovics J., Katona G.O.H., The poset of closures as a model of changing databases, Order, (1987), 127-142. 
6. Demetrovics J., Logical and structural Investigation of Relational Datamodel (Hungarian language). MTA-SZTAKI Tanulmanyok, Budapest, 114 (1980), 1-97

7. Demetrovics J., Thi V. D., Some results about functional dependencies, Acta Cybernetica, VIII/3 (1988), 273-278.

8. Demetrovics J., Thi V.D., Relational keys, Acta Cybernetica, VIII/3 (1988), 279-285.

9. Demetrovics J., Thi V.D., On Keys in the Relational Datamodel, EIK, 24 (1988), 515-519.

10. Gottlob G., Libkin L., Investigations on Armstrong relations, dependency inference, and excluded functional dependencies, Ata Cybernetica, Tom. 9, Fasc. 4 (1990), 385-402.

11. Lucchesi C. L., Osborn S. L., Candidate keys for relation, J. Comput. Syst. Scien., 17, 2 (1978), 270-279.

12. Mannila H., Raiha K. J., Design by Example: An Application of Armstrong relations, J. Comput. Syst. Scien., 33 (1986), 126-141.

13. Mannila H., Raiha K. J., Algorithm for inferring functional dependencies, Proc. of the thirteenth international conference on very large data bases, Brighton, September 1987.

14. Thi V.D., Investigation on Combinatorial Characterizations Related to Functional Dependency in the Relational Datamodel (Hungarian language). MTA-SZTAKI Tanulmanyok, Budapest, 191 (1986), 1-157. Ph. D. Dissertation.

15. Thi V. D., Minimal keys and Antikeys, Acta Cybernetica, Tom. 7, Fasc. 4 (1986), 361-371.

16. Thi V.D., On Antikeys In The Relational Datamodel (Hungarian language), Alkalmazott Matematikai Lapok, 12 (1986), 111-124.

17. Demetrovics J., Henesey G., Libkin L. O., Muchnik I. B., Normal form relation schemes: a new characterization, Acta Cybernetica, 10, 3 (1992), 141-153.

Institute of Information Technology

National Centre for Science and Technology of Vietnam. 